\title{
Comparación del éxito anatómico y funcional del tratamiento del desprendimiento de retina regmatógeno con afección macular entre dos técnicas de retinopexia
}

\author{
Comparison of anatomic and functional success of treatment for retinal detachment with \\ macular involvement between two retinopexy techniques
}

\author{
Paola Brito-Sandoval', Itzel C. Espinosa-Soto ${ }^{1}$, Juan A. Ramírez-Estudillo y Virgilio Lima-Gómez* \\ ${ }^{1}$ Servicio de Retina y Vítreo, Fundación Hospital Nuestra Señora de la Luz, I.A.P.; ${ }^{2}$ Servicio de Oftalmología, Hospital Juárez de México. Ciudad \\ de México, México
}

\begin{abstract}
Resumen
Objetivo: Comparar las frecuencias de éxito anatómico y funcional en ojos con desprendimiento de retina regmatógeno no complicado tratados con exoplante o vitrectomía. Método: Estudio observacional, retrospectivo, comparativo y longitudinal, en pacientes con desprendimiento de retina regmatógeno, agudeza visual $<20 / 200$ y afección macular, tratados con exoplante (grupo 1) o vitrectomía (grupo 2), y seguimiento por 9 meses, sin vitreorretinopatía proliferativa ni otras enfermedades vitreorretinianas que redujeran la visión. Se compararon las frecuencias de éxito anatómico (retina aplicada) y funcional (agudeza visual > 20/200) al final del seguimiento (ji al cuadrado y razón de momios). Resultados: 114 pacientes, edad promedio $48.5 \pm 16.6$ años, duración promedio del desprendimiento $49.8 \pm 32.0$ días, 33 casos en el grupo 1 (28.9\%) y 81 en el grupo 2 (71.1\%). Hubo éxito anatómico en 27 casos del grupo 1 (81.8\%; intervalo de confianza del 95\% [IC 95\%]: 68.7-95.0) y en 74 del grupo 2 (91.4\%; IC 95\%: 85.2-97.5; $p=0.1)$; 14 casos del grupo 1 (42.4\%) y 21 del grupo 2 (25.9\%) tuvieron éxito funcional $(p=0.1)$. Discusión: En nuestro medio, las frecuencias de éxito anatómico y funcional después de tratar el desprendimiento de retina regmatógeno no difieren entre el exoplante y la vitrectomía. Debe favorecerse la detección temprana para aumentar la frecuencia de mejoría visual posoperatoria.
\end{abstract}

PALABRAS CLAVE: Desprendimiento de retina. Exoplante. Mácula. Retinopexia. Vitrectomía.

\begin{abstract}
Objective: To compare the frequency of anatomic and functional success, between eyes with uncomplicated rhegmatogenous retinal detachment treated with exoplant or vitrectomy. Method: Non-experimental, retrospective, comparative and longitudinal study in patients with rhegmatogenous retinal detachment, <20/200 visual acuity and macular involvement, treated with exoplant (group 1) or vitrectomy (group 2), and 9 months follow up, without proliferative vitreoretinopathy or other vitreoretinal disease that reduced vision. The frequencies of anatomic success (attached retina) and functional success (visual acuity > 20/200) were compared between groups at the end of follow up (chi squared and odds ratio). Results: 114 patients, mean age $48.5 \pm 16.6$ years, mean detachment duration $49.8 \pm 32.0$ days; group 1 had 33 subjects (28.9\%) and group 2 had 81 (71.1\%). Anatomic success was achieved in 27 subjects of group 1 (81.8\%; 95\% confidence interval [95\% Cl]: 68.7-95) and in 74 of group 2 (91.4\%; 95\% Cl: 85.2-97.5; $p=0.1)$; 14 subjects of group 1 (42.4\%) and 21 of group 2 (25.9) had functional success
\end{abstract}

\author{
Correspondencia: \\ *Virgilio Lima Gómez \\ Avda. Instituto Politécnico Nacional, 5160 \\ Col. Magdalena de las Salinas, Del. Cuauhtémoc \\ C.P. 07760 , Ciudad de México, México \\ E-mail: forscher7@gmail.com
}

Fecha de recepción: 27-04-2018

Fecha de aceptación: 23-06-2018

DOI: $10.24875 / C I R U .18000405$
Cir Cir. 2019;87:136-140

Contents available at PubMed www.cirugiaycirujanos.com 
$(p=0.1)$. Discussion: In our media, the frequencies of anatomic and functional success after treating rhegmatogenous retinal detachment did not differ between exoplant and vitrectomy. Early detection should be favored, to increase the frequency of postoperative visual improvement.

KEY WORDS: Retinal detachment. Exoplant. Macula. Retinopexy. Vitrectomy.

\section{Introducción}

En el desprendimiento de retina, la porción neurosensorial de esta estructura se separa del epitelio pigmentario, lo cual reduce la agudeza visual'; el desprendimiento puede ser regmatógeno, por tracción, seroso o mixto. El primero es el más común y se presenta cuando un agujero en la retina permite que pase líquido del vítreo al espacio subretiniano; su incidencia estimada en el mundo es de 6.3 a 17.9 casos por cada 100,000 pacientes al año $0^{2,3}$, y aumenta en ojos miopes ${ }^{4} 0$ después de cirugía de catarata ${ }^{5}$.

El desprendimiento regmatógeno requiere cirugía (retinopexia), y las técnicas más usadas son el exoplante escleral/cerclaje y la vitrectomía, que reducen la tracción vítrea que mantiene abierto el agujero o desgarro en la retina, lo cual permite cerrarlo y drenar el líquido subretiniano. El exoplante limita la tracción al indentar el sector donde está el agujero retiniano o los $360^{\circ}$ de la esclera (cerclaje), y la vitrectomía es un procedimiento intraocular en el cual se retira el vítreo que causa tracción. Aunque los equipos para vitrectomía han evolucionado más que los exoplantes, el éxito anatómico con ambas técnicas es cercano al 90\% ${ }^{6}$. La vitreorretinopatía proliferativa es la principal causa de falla anatómica ${ }^{7}$, mientras que la afección de la mácula, la agudeza visual y el tiempo de evolución preoperatorios limitan la recuperación funcional ${ }^{8}$.

Los desenlaces anatómico y visual dependen de la cirugía oportuna y de elegir la técnica adecuada. Aunque la vitrectomía se realiza más comúnmente ${ }^{9}$, en nuestro medio no existe información que indique que sus resultados superen a los de un exoplante para tratar los casos más frecuentes, atendidos tardíamente y con afección macular. Se realizó un estudio para comparar la proporción de éxito anatómico y funcional en los ojos con desprendimiento de retina regmatógeno primario no complicado tratados con exoplante o con vitrectomía.

\section{Método}

Se desarrolló un estudio observacional, retrospectivo, comparativo y longitudinal, en pacientes tratados quirúrgicamente por desprendimiento de retina regmatógeno en un hospital oftalmológico de referencia. Se evaluaron pacientes intervenidos entre el 1 de septiembre de 2013 y el 30 de septiembre de 2014; el estudio se efectuó del 1 de junio al 31 de octubre de 2015, se apegó a los principios de la declaración de Helsinki y fue autorizado por las comisiones de investigación y ética en investigación del hospital donde se realizó.

Se incluyeron pacientes de ambos sexos, con edades de 6 a 80 años, con desprendimiento de retina regmatógeno tratado por primera vez en la institución con un solo procedimiento quirúrgico, sin complicaciones, con agudeza visual corregida $<20 / 200$ al momento del diagnóstico, fáquicos, con inclusión de la mácula en el desprendimiento y seguimiento mínimo de 9 meses. Se excluyeron los pacientes que antes de la cirugía o durante el seguimiento presentaron vitreorretinopatía proliferativa o cualquier otra enfermedad vitreorretiniana que pudiera modificar el resultado quirúrgico anatómico o funcional final.

La variable independiente fue la técnica quirúrgica empleada para tratar el desprendimiento de la retina. Se definió operativamente como la intervención o intervenciones requeridas para aplicar la retina durante el transoperatorio. Se trató de una variable cualitativa nominal, calificada como exoplante cuando solo se empleó esa técnica quirúrgica en forma circunferencial (cerclaje) o localizada (grupo 1), y como vitrectomía cuando se empleó ese procedimiento intraocular, con independencia de las técnicas adicionales utilizadas, incluido el cerclaje (grupo 2).

Las variables dependientes fueron el éxito anatómico, definido operativamente como presente cuando la retina estaba aplicada 9 meses después de la cirugía, y el éxito funcional, definido operativamente como una agudeza visual corregida posoperatoria $>20 / 200$ al final del seguimiento. Se consideraron variables secundarias la edad, el sexo, la presencia de miopía $>-6.0$ dioptrías, la etiología traumática del desprendimiento de la retina y el tiempo de evolución del desprendimiento al momento de la cirugía.

Se identificaron la proporción y los intervalos de confianza del 95\% (IC 95\%) de los ojos con éxito 
Tabla 1. Comparación de las variables basales entre grupos

\begin{tabular}{lccc}
\hline Variable & $\begin{array}{c}\text { Grupo 1 } \\
(\mathbf{n}=\mathbf{3 3})\end{array}$ & $\begin{array}{c}\text { Grupo 2 } \\
(\mathbf{n}=\mathbf{8 1})\end{array}$ & $\mathbf{p}$ \\
\hline Edad (años) & $44.1 \pm 16.3$ & $50.3 \pm 16.4$ & $0.07^{*}$ \\
Sexo femenino & $33.3 \%$ & $54.3 \%$ & $0.04^{\dagger}$ \\
Miopía & $27.3 \%$ & $25.9 \%$ & $0.9^{\dagger}$ \\
Trauma & $12.1 \%$ & $11.1 \%$ & $0.9^{\dagger}$ \\
Tiempo de evolución (días) & $41.2 \pm 20.6$ & $53.3 \pm 35.2$ & $0.07^{\star}$
\end{tabular}

${ }^{*}$ de Student para medias independientes.

†Ji al cuadrado.

Tabla 2. Resultado anatómico y funcional de la cirugía, por grupo

\begin{tabular}{lccc}
\hline Resultado & Grupo 1 $(\mathbf{n}=\mathbf{3 3})$ & Grupo 2 $(\mathbf{n}=\mathbf{8 1})$ & $\mathbf{p}^{*}$ \\
\hline Éxito anatómico & $27(81.8 \%)$ & $74(91.4 \%)$ & 0.1 \\
Éxito funcional & $14(42.4 \%)$ & $21(25.9 \%)$ & 0.1 \\
\hline *Ji al cuadrado. & & &
\end{tabular}

anatómico y con éxito funcional en cada grupo; las proporciones se compararon entre grupos mediante la prueba de ji al cuadrado y la razón de momios. Adicionalmente se comparó la distribución de las variables secundarias entre los grupos, con la prueba de ji al cuadrado para las variables cualitativas y la prueba t de Student para muestras independientes en el caso de las variables cuantitativas.

Se consideró significativo un valor de $p<0.05$. La información se almacenó y analizó con la versión 21 del programa SPSS para Windows. Toda la información se obtuvo de los registros en los expedientes, por lo que generó un riesgo menor al mínimo. Se protegió la confidencialidad de los sujetos en estudio.

\section{Resultados}

Se revisaron 114 pacientes con edades de 6 a 80 años (48.5 \pm 16.6$), 55$ de sexo femenino (48.2\%), 30 presentaban miopía (26.3\%) y 13 tenían antecedente de trauma (11.4\%). El tiempo de evolución del desprendimiento fue de 21 a 150 días $(49.8 \pm 32.0)$. Treinta y tres ojos se asignaron al grupo $1(28.9 \%)$ y 81 al grupo 2 (71.1\%). La comparación de las variables secundarias entre ambos grupos se presenta en la tabla 1.

Se obtuvo éxito anatómico en 101 casos (88.6\%; IC 95\%: 82.8-94.4): 27 del grupo 1 y 74 del grupo 2. La proporción de ojos con este desenlace en el grupo 1 (81.8\%; IC 95\%: 68.7-95.0) no difirió de la encontrada en el grupo 2 (91.4\%; IC 95\%: 85.2-97.5) (Tabla 2). De los 74 pacientes del grupo 2 que tuvieron éxito anatómico, $46(62.2 \%)$ requirieron tamponamiento con aceite de silicón.

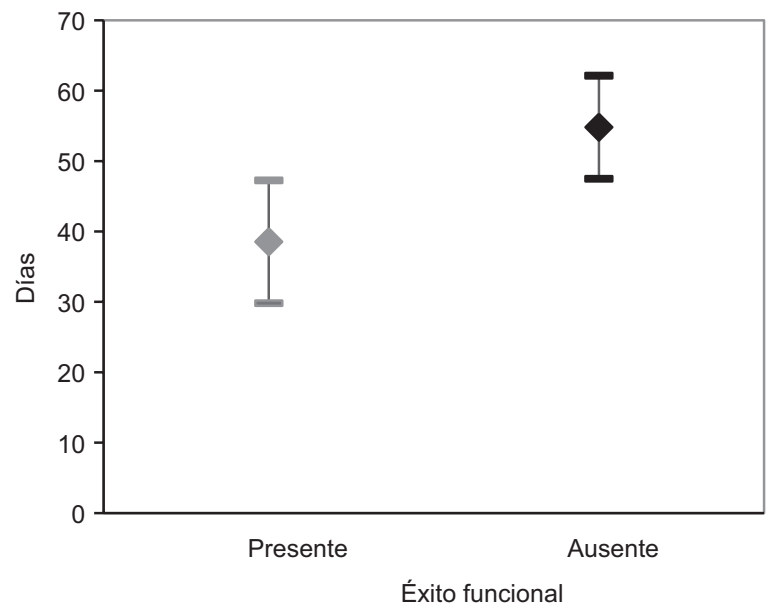

Figura 1. Tiempo de evolución del desprendimiento de retina en ojos con o sin éxito funcional (promedio, intervalos de confianza del 95\%).

Tuvieron éxito funcional 35 ojos (30.7\%; IC 95\%: 22.2-39.2): 14 del grupo 1 (42.4\%; IC 95\%: 25.6-59.3) y 21 del grupo 2 (25.9\%; IC 95\%: 16.4-35.5; $p=0.1)$. De los 21 ojos del grupo 2 con éxito funcional, $11(52.3 \%)$ requirieron tamponamiento con aceite de silicón. La proporción de éxito funcional no difirió entre los ojos con éxito anatómico $(31 / 101,30.7 \%)$ y los que no lo alcanzaron $(4 / 13,30.8 \% ; p=0.9)$.

El tiempo de evolución del desprendimiento en los ojos con éxito funcional fue de $38.5 \pm 26.3$ días, que fue menor al de los ojos que no alcanzaron esa función $(54.8 \pm 33.2$ días; $p=0.006)$ (Fig. 1). No se identificó ningún punto de corte temporal a partir del cual la probabilidad de alcanzar éxito funcional fuera mayor. Sin embargo, en el grupo 1 el tiempo promedio de evolución en los ojos con éxito funcional fue de $31.2 \pm 9.5$ días, y en los que no lo alcanzaron fue de $48.6 \pm 23.5$ días; en el grupo 2, el tiempo de evolución no difirió entre los ojos con éxito funcional y sin él $(p=0.007)$ (Fig. 2).

En los ojos con evolución hasta 40 días hubo una mayor proporción de casos en los que se alcanzó éxito funcional con agudeza visual > 20/200 en el grupo 1 (12/21, 57.1\%) que en el grupo 2 (14/44, $31.8 \% ; p=0.51)$, lo cual marcó una tendencia estadística (prueba exacta de Fisher para una cola: 0.047).

\section{Discusión}

El éxito anatómico después de la cirugía para tratar el desprendimiento de retina primario en ojos con afección de la mácula fue del $88.6 \%$, y la proporción de ojos que presentaron este resultado no varió entre los que se trataron con cerclaje/exoplante y los que se trataron con vitrectomía. La proporción de éxito 


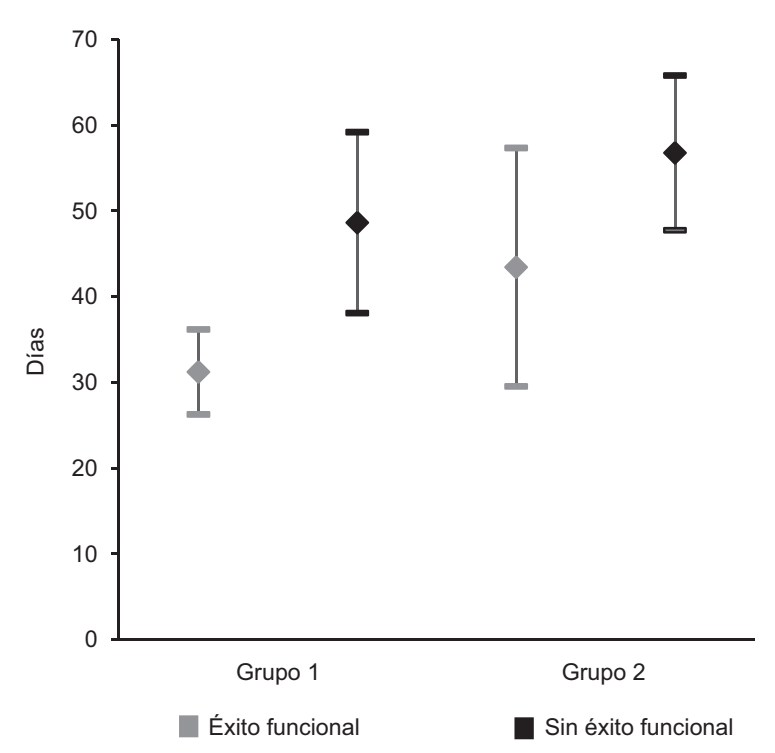

Figura 2. Comparación del tiempo de evolución del desprendimiento en ojos con y sin mejoría visual, en ambos grupos (promedio, intervalos de confianza del 95\%).

funcional, de acuerdo con la definición operativa, tampoco difirió entre ambos grupos.

La proporción de éxito anatómico fue semejante a la reportada internacionalmente $(83.1-86 \%)^{10-12}$ en forma global y para cada técnica (exoplante $86 \%$ y vitrectomía $90 \%)^{13}$. Aunque ya se ha reportado que el promedio de agudeza visual mejora en los pacientes con afección macular ${ }^{14}$, no se había determinado en nuestro medio la proporción de pacientes que alcanza esa mejoría. En el estudio de Doyle, et al. ${ }^{15}$, los pacientes con agudeza visual preoperatoria $<20 / 200$ tuvieron un promedio de agudeza visual posoperatoria de 20/80, pero la mediana de duración del desprendimiento con afección macular fue de 9 días,mientras que en este estudio ningún paciente se trató antes de 20 días.

Llama la atención que el tiempo de evolución haya sido menor en los ojos que alcanzaron una agudeza visual $>20 / 200$ solo cuando se trataron con exoplante; esta diferencia podría deberse a un sesgo de selección que hubiera asignado ojos con menor extensión o elevación del desprendimiento al tratamiento temprano con exoplante, pero se requeriría contar con la descripción de esas características en todos los ojos para hacer una comparación adecuada.

Se observa una tendencia estadística para alcanzar una mayor proporción de ojos con agudeza visual $>20 / 200$ en los casos tratados antes de 40 días, que deberá reevaluarse. Aunque el resultado quirúrgico no sea óptimo, debe tomarse en cuenta que la atención fue tardía para la mayoría de los ojos, y que la afección de la mácula sugería que la probabilidad de alcanzar alguna mejoría visual era baja.

Una potencial limitación del estudio es que la asignación de la técnica quirúrgica no fue aleatoria, lo cual genera sesgos relacionados con la experiencia del cirujano para tratar casos de distinta complejidad. Otra limitación es la falta de descripción de las características del desprendimiento, como la elevación de la mácula (medida por tomografía de coherencia óptica $)^{16}$, la extensión del desprendimiento ${ }^{17,18}$, el tipo de defecto retiniano que lo ocasionó y su localización ${ }^{19}$. Aunque la inclusión de la mácula en el desprendimiento empeore el pronóstico, obtener una mejoría visual hasta en el $39.2 \%$ de los casos requiere evaluar otras variables asociadas con ese desenlace. Wong, et al. ${ }^{20}$ reportaron una proporción de mejoría semejante (43.2\%) en ojos cuyo desprendimiento afectaba la mácula tratados con exoplante, pero su criterio de éxito funcional fue una agudeza visual $\geq 20 / 40$.

También sería conveniente estratificar el análisis conforme al tamponamiento intraocular empleado durante la vitrectomía; aunque la decisión puede cambiar durante la cirugía, para fines de investigación es preferible estandarizar, de manera que al realizar el análisis se cuente con grupos más homogéneos.

En resumen, el éxito anatómico y funcional del desprendimiento de retina regmatógeno primario en nuestro medio, con afección macular y capacidad visual < 20/200, no difirió entre los ojos tratados con vitrectomía y los tratados mediante exoplante. Deberá favorecerse la referencia temprana e identificar otras características sobre las que pueda intervenirse para aumentar la proporción de ojos con mejoría visual posoperatoria.

\section{Conflicto de intereses}

El estudio fue financiado por la Fundación Hospital Nuestra Señora de la Luz, I.A.P. Los autores declaran que no tuvieron conflicto de intereses para el desarrollo del estudio, ni lo tienen para la publicación del trabajo.

\section{Responsabilidades éticas}

Protección de personas y animales. Los autores declaran que para esta investigación no se han realizado experimentos en seres humanos ni en animales. 
Confidencialidad de los datos. Los autores declaran que han seguido los protocolos de su centro de trabajo sobre la publicación de datos de pacientes.

Derecho a la privacidad y consentimiento informado. Los autores declaran que en este artículo no aparecen datos de pacientes.

\section{Bibliografía}

1. Ho VY, Wehmeier JM, Shah GK. Wide-field infrared imaging. A descriptive review of characteristics of retinoschisis, retinal detachment and schisis detachments. Retina. 2016;36:1439-45.

2. Mitry D, Charteris DG, Fleck BW, Campbell H, Singh J. The epidemiology of rhegmatogenous retinal detachment: geographical variation and clinical associations. Br J Ophthalmol. 2010;94:678-84.

3. Chen SN, Lian IB, Wei YJ. Epidemiology and clinical characteristics of rhegmatogenous retinal detachment in Taiwan. $\mathrm{Br} J$ Ophthalmol. 2016;100:1216-20.

4. Kang HM, Lee CS, Park HJ, Lee KH, Byeon SH, Koh HJ, et al. Characteristics of rhegmatogenous retinal detachment after refractive surgery: comparison with myopic eyes with retinal detachment. Am J Ophthalmol. 2014;157:666-72.

5. Bjerrum SS, Mikkelsen KL, La Cour M. Risk of pseudophakic retinal detachment in 202,226 patients using the fellow nonoperated eye as reference. Ophthalmology. 2013;120:2573-9.

6. Schaal S, Sherman MP, Barr CC, Kaplan HJ. Primary retinal detachment repair. Comparison of 1-year outcomes of four surgical techniques. Retina. 2011;31:1500-4.

7. Adelman RA, Parnes AJ, Michalewska Z, Ducournau D. The European Vitreo-Retinal Society (EVRS) Retinal Detachment Study Group. Clinical variables associated with failure of retinal detachment repair. The European Vitreo-Retinal Society detachment study report number 4. Ophthalmology. 2014;121:1715-9.

8. Kobayashi M, Iwase T, Yamamoto K, Ra E, Murotani K, Matsui S, et al. Association between photoreceptor regeneration and visual acuity fo- llowing surgery for rhegmatogenous retinal detachment. Invest Ophthalmol Vis Sci. 2016;57:889-98.

9. Hang JC. Regional practice patterns for retinal detachment repair in the United States. Am J Ophthalmol. 2012;153:1125-8.

10. Adelman RA, Parnes AJ, Ducournau D, the European Vitreo-Retinal Society (EVRS) Retinal Detachment Study Group. Strategy for the management of uncomplicated retinal detachments. The European Vitreo-Retinal Society Retinal Detachment Study Report 1. Ophthalmology. 2013;120:1804-8.

11. Rush R, Simunovic MP, Sheth S, Chang A, Hunyor AP. 23-gauge pars plana vitrectomy versus scleral buckling versus combines pars plana vitrectomy-scleral buckling for medium complexity retinal detachment repair. Asia-Pac J Ophthalmol. 2014;3:215-9.

12. Al-Hinai AS, Al-Abri MS. Outcome of rhegmatogenous retinal detachment repair: experience of a tertiary center in Oman. Oman $\mathrm{J}$ Ophthalmol. 2013;6:179-82.

13. Schaal S, Sherman MP, Barr CC, Kaplan HJ. Primary retinal detachment repair. Comparison of 1-year outcomes of four surgical techniques. Retina. 2011;31:1500-4.

14. van de Put MAJ, Croonen D, Nolte IM, Japing WJ, Hooymans JMM, Los LI. Postoperative recovery of visual function after macula-off rhegmatogenous retinal detachment. PLoS One. 2014;9:e99787.

15. Doyle E, Herbert EN, Bunce C, Williamson TH, Laidlaw DAH. How effective is macula-off retinal detachment surgery? Might good outcome be predicted? Eye. 2007;21:534-40.

16. Cho M, Witmer MT, Favarone G, Chan RVP, D'Amico DJ, Kiss S. Optical coherence tomography predicts visual outcome in macula-involving rhegmatogenous retinal detachment. Clin Ophthalmol. 2012;6:91-6.

17. Wicham L, Bunce $C$, Wong D, Charteris DG. Retinal detachment repair by vitrectomy: simplified formulae to estimate the risk of failure. $\mathrm{Br} \mathrm{J}$ Ophthalmol. 2011;95:1239-44.

18. Mitry D, Awan MA, Borooah S, Rehman Siddiqui MA, Brogan K, Fleck BW, et al. Surgical outcome and risk stratification for primary retinal detachment repair: results from the Scottish Retinal Detachment Study. Br J Ophthalmol. 2012;96:730-4.

19. Noori J, Bilonick RA, Eller AW Scleral buckle surgery for primary retinal detachment without posterior vitreous detachment. Retina. 2016;36: 2066-71.

20. Wong CW, Yeo IY, Loh BK, Wong EY, Wong DW, Ong SG, et al. Scleral buckling versus vitrectomy in the management of macula-off primary rhegmatogenous retinal detachment. Retina. 2015.35:2552-7. 